\title{
Grocery Distribution with Multi-Compartment Vehicles
}

\author{
Manuel Ostermeier ${ }^{1}$
}

\begin{abstract}
This article discusses the use of multi-compartment vehicles in grocery distribution. In grocery distribution, special characteristics of available products, i.e., nutritions in particular, have to be considered for transportation. Retailers offer a wide range of products from frozen over fresh to ambient goods. The distribution therefore asks for specialized vehicles that offer the required temperature control. This can be addressed with single-compartment vehicles, each dedicated to exactly one temperature, or multi-compartment vehicles (MCVs). Multi-compartment vehicles offer the possibility to separate the loading area of the vehicle into multiple chambers (compartments), each with a different temperature setting. The implication of this multiple compartments for the use in distribution, the technical configuration possibilities of MCVs, and both advantages and disadvantages are discussed in detail in this article. Further, current literature that addresses the use of MCV in the field of grocery distribution is presented. The corresponding publications and their research topic are summarized and the main findings are given. In this way, this article provides a detailed overview of the use of MCVs in grocery retailing and the relevant problems discussed in literature.
\end{abstract}

\section{Introduction}

Retailing is a highly dynamic and competitive field of $\mathrm{b}$ usiness. In g eneral, $\mathrm{r}$ etailers have to cope with many challenges due to the fast development over the past decades. Nowadays every retailer has to face and follow numerous trends and developments. By way of example, the rise of on-line retailers and the arising task of multi-channel retailing require a permanent adjustment of a company's strategy. Retailers are obliged to find answers to these n ew trends in order to compete on the market.

Despite all these new challenges for retailers, one of the core problems for each company is the definition and optimization of an efficient supply c hain. As such, all entities of the supply chain have to be streamlined and each entity needs to align its processes to the overall success of the company. An essential part of a well functioning supply chain is the distribution of goods between the single chain links. This is particularly true for the distribution of groceries from the distribution center

\footnotetext{
${ }^{\text {I}}$ Catholic University Eichstätt-Ingolstadt, Supply Chain Management \& Operations, Auf der Schanz 49, 85049 Ingolstadt, Germany, manuel.ostermeier@ku.de
} 
(DC) to the stores and therefore the last link of the chain. The distribution of groceries comprises the planing and operating of efficient delivery tours to the customers. These kinds of delivery problems are denoted as vehicle routing problems (VRP) in literature. For a general overview of VRP the works of Toth and Vigo [2014], Laporte [2009] and Golden et al. [2008] are recommended.

In this article we will consider the distribution of groceries with a detailed description of the transportation requirements and the use of suitable vehicles for the distribution fleet. In particular, multi-compartment vehicles (MCV) are introduced as an efficient new technology for the transportation of products with different temperature requirements and the corresponding VRP with MCV will be highlighted. MCVs are able to combine several temperature-specific goods in their loading area as they are able to split it in different, separated and isolated chambers. They constitute an attractive alternative to single-compartment vehicles (SCV) that have been used as a standard for distribution in the past.

In the remainder of this article the overall requirements for distribution will be explained, followed by a detailed description of MCVs, SCVs and their role in grocery distribution. Further, in Section 2 we will detail current research on the use of MCVs in grocery distribution. This involves new challenges that arise when MCVs are used for distribution, like additional costs and handling process, as well as extended planing problems for the definition of delivery tours.

\subsection{Distribution Requirements in Retailing}

The distribution of groceries to customers starts at a regional or central DC. In the DC all products for the customers assigned to the corresponding DC are consolidated and from there the last mile transportation starts, i.e., the supply of stores. Goods of all categories (e.g., frozen, fresh and ambient) are stored at the same DC but require different temperature regulations for stocking. A separate section is therefore required for each temperature zone at the DC. We denote all products that have similar temperature requirements and can therefore be stored in the same area of the DC as one product segment, i.e., each product segment stands for one section in the DC. In Figure 1 an exemplary layout of an DC divided into several areas according to the stored segments is presented.

The definition of product segments is highly dependent on the retailer and its product variety. By way of example, a Portuguese retailer might consider fresh fish as a separate segment as it shows specific temperature requirements and needs to be treated separately due to the characteristic smell. Figure 2 illustrates five possible product segments that occur in grocery distribution and can be seen as standard for large retailers.

Besides the storage also the distribution process itself is influenced by the separation of products 


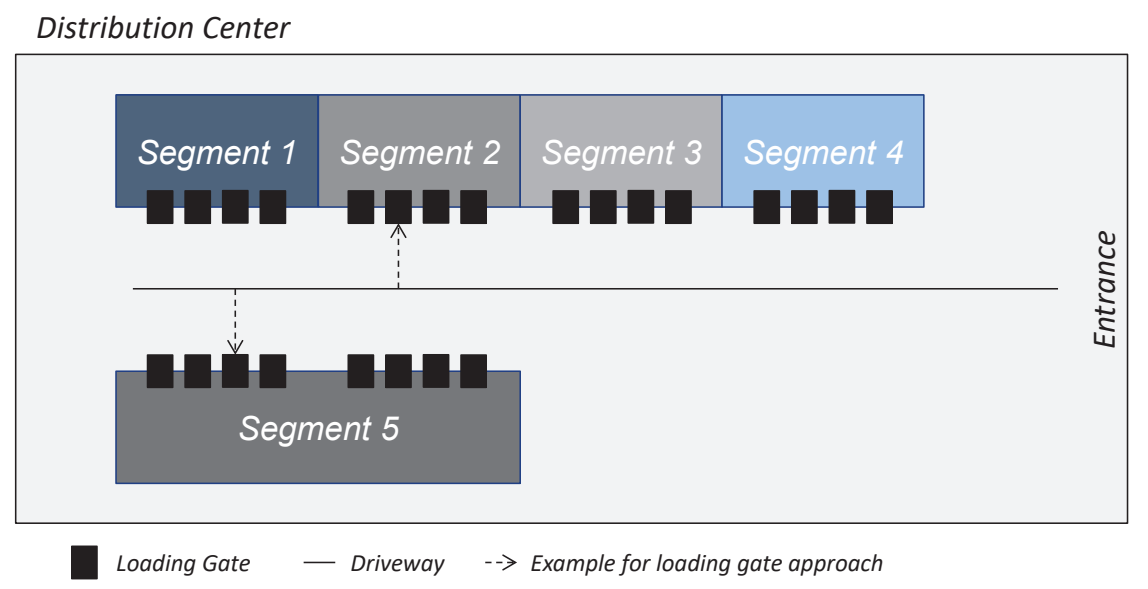

Figure 1: Exemplary DC layout

into different temperature categories. In most VRPs only one delivery per customer is regarded. Usually, different items/orders are summarized in one delivery and each customer is approach at most once each day. In the field of grocery distribution one order comprises one product segment only. As each product segment has specific temperature requirements, the combination of different orders in one delivery is not as simple as in other VRP variants, as for instance the delivery of mail or wood. If multiple segments and therefore orders cannot be combined, a customer has to be approached several times to deliver all demanded goods. However, a combination of segments is possible if MCVs are used. Retailers can therefore choose between SCVs and MCVs for their distribution fleet. In the following, we will have a detailed look at the attributes of the different vehicle types and their use in distribution.

\begin{tabular}{|c||c|c|c|c|c|}
\hline $\begin{array}{c}\text { Product } \\
\text { segment }\end{array}$ & $\begin{array}{c}\text { Deep } \\
\text { frozen }\end{array}$ & $\begin{array}{c}\text { Meat and } \\
\text { sausages }\end{array}$ & $\begin{array}{c}\text { Vegetables } \\
\text { and fruits }\end{array}$ & $\begin{array}{c}\text { Diary } \\
\text { products }\end{array}$ & Ambient \\
\hline \hline Temperature & $-18^{\circ} \mathrm{C}$ & $+2^{\circ}$ to $+6^{\circ} \mathrm{C}$ & $+4^{\circ}$ to $+6^{\circ} \mathrm{C}$ & $+6^{\circ} \mathrm{C}$ & No regulation \\
\hline
\end{tabular}

Figure 2: Example of products segments in grocery distribution (temperature in degree Celsius)

\subsection{Single- and Multi-Compartment Vehicles}

Single-Compartment Vehicles. As stated above, SCVs have been used as a standard transportation vehicles for grocery distribution over the last decades. An SCV is able to transport one temperature zone at a time and uses the complete loading area for this purpose. Usually, there are two types of SCVs for grocery distribution: SCVs with a cooling aggregate to transport either fresh or frozen goods and SCVs without cooling for ambient products. The loading and unloading happens from the rear of the truck and the loading area can only be accessed through the gate at the rear. Figure 3 displays the layout of an SCV. 


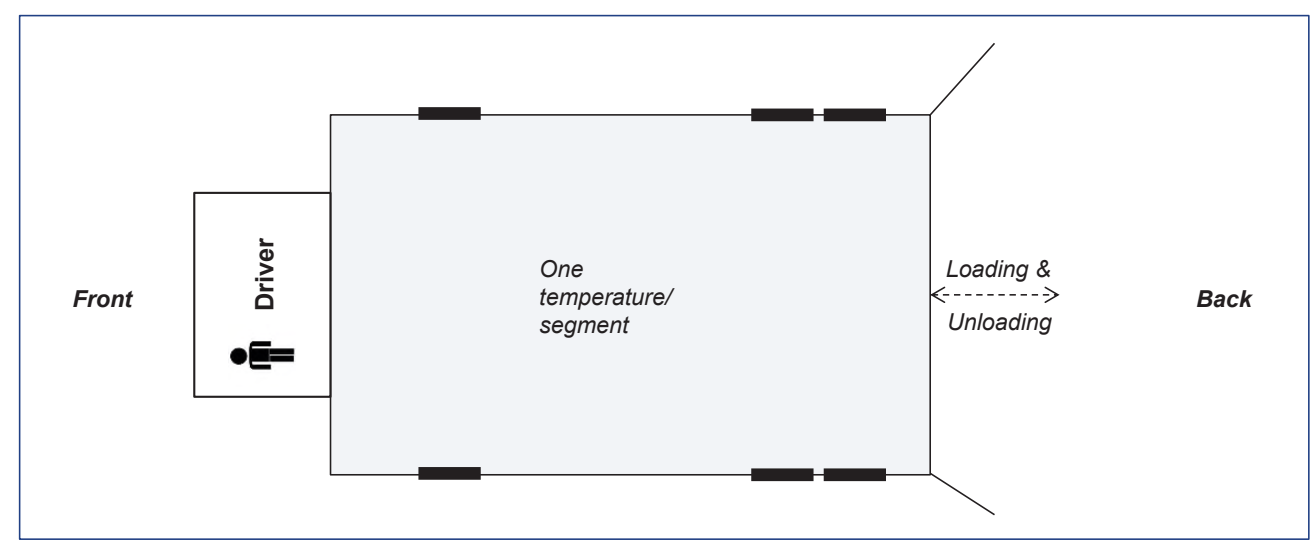

Figure 3: Sketch of a single-compartment vehicle

Multi-Compartment Vehicles. Additional to SCVs, the technical more advanced MCVs can be used for transportation in grocery distribution. The use of MCVs is significantly increasing over the last years and many retailers already rely on their higher flexibility. MCVs have been developed to enable the joint transportation of multiple product segments and therefore temperature zones on the same vehicle. The main characteristic that distinguishes MCVs from SCVs is, as the name suggests, the ability to separate the loading area into multiple compartments or chambers. Each of those chambers can be dedicated to one specific temperature zone. They are separated of from other chambers using longitude separation walls and flexible doors for each chamber. With this, the size and also the number of compartments is completely flexible. In practice, up to five different compartments can be setup on a single vehicle. There is no loss in capacity if multiple chambers are used due to the flexible closing of compartments. A single compartment can have the size of the full truck capacity which is than equal to the use of a SCV, or a single transportation unit (e.g. the size of one pallet or role cage).

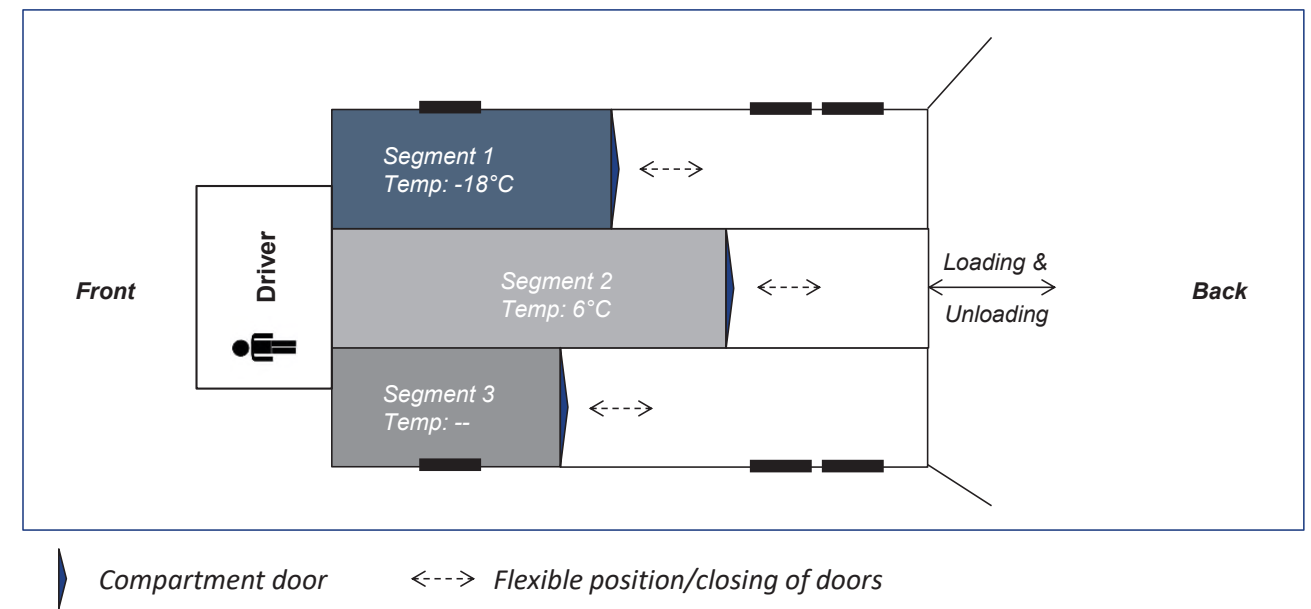

Figure 4: Sketch of a multi-compartment vehicle 
Figure 4 illustrates the layout of an MCV. The general setup and properties of MCVs are equal to those of SCVs. There is no differentiation in capacity and further, the loading and unloading of products happens from the rear of each truck. As a consequence, both vehicle types have to approach the loading gates at the DC or the loading area at stores backwards. An overview of the overall distribution process and the special characteristics of SCVs and MCVs for the distribution are detailed next.

\subsection{The Distribution Process}

The distribution process in grocery distribution requires the consideration of several specific characteristics of SCVs and MCVs. Some parts of the distribution are the same for both vehicles types and others need only to be considered for the corresponding type. The similarities and differences are explained in detail in the subsequent paragraphs.

Distribution Similarities for SCVs and MCVs. As stated above, some characteristics of the distribution process are the same if SCVs or MCVs are used. The capacity available on each vehicle is the same, only dependent on the vehicle size but not on the type. Further, both types are loaded and unloaded from the rear of the truck and the processes involved for the loading and unloading, i.e., the moving of pallets and/or role cages to/from the trucks are equivalent. As trucks are accessed from the rear, each vehicle type has to approach the loading gate at the DC or at the customer store driving backwards. Besides this similarities, the individual distribution process is detailed for SCVs and MCVs.

The Distribution Process with SCVs. When SCVs are used for transportation, each vehicle is dedicated to one product segment, i.e., one temperature zone. As a consequence, an SCV approaches one loading gate at the distribution center to load all orders of the corresponding segment. The delivery of this segment is than carried out, supplying one or several customers with the corresponding products. Naturally, each costumer on the route is supplied with the same segment and only customers with demand for the loaded segment can be included in an SCV tour. If a customer has demand for another segment on the same delivery day, a second SCV tour has to be defined to deliver products of the second category. The loading situation for SCVs is illustrated in Figure 5. It shows the delivery of two different segments with SCVs.

The Distribution Process with MCVs. The use of MCVs results in various changes in the distribution process. The main characteristic for MCVs is the combination of different product segments on the loading area by setting up different compartments. As multiple segments are delivered jointly, also multiple areas in the DC have to be approached to load the corresponding segments. Hence, if four 

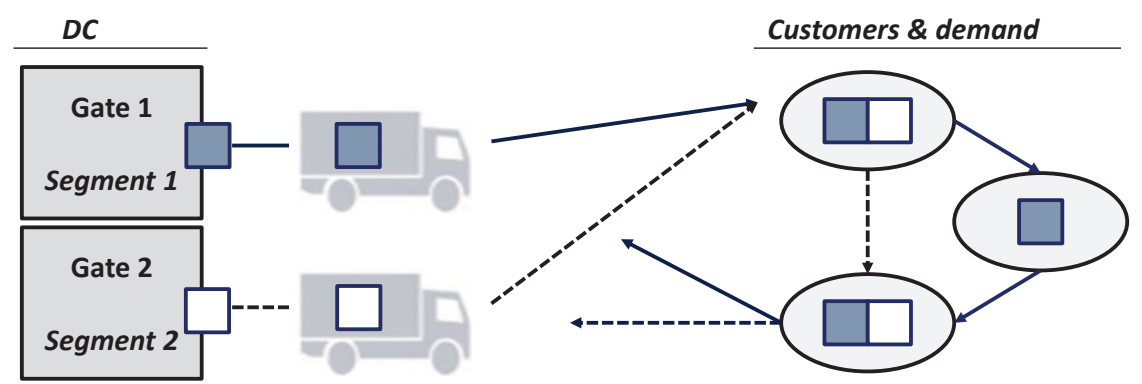

Figure 5: Distribution Process for SCVs

segments are transported by one MCV, also four loading gates at the DC have to be approached. This leads to a more complex and therefore more time intensive loading process at DCs. When an MCV approaches a loading gate for one segment, all corresponding orders of the segment have to be loaded and therefore the setup of compartments has to be known beforehand. Multiple approaches of the same segment are not feasible as this would lead to an unmanageable planning situation at the DC and an significant increase in loading times.
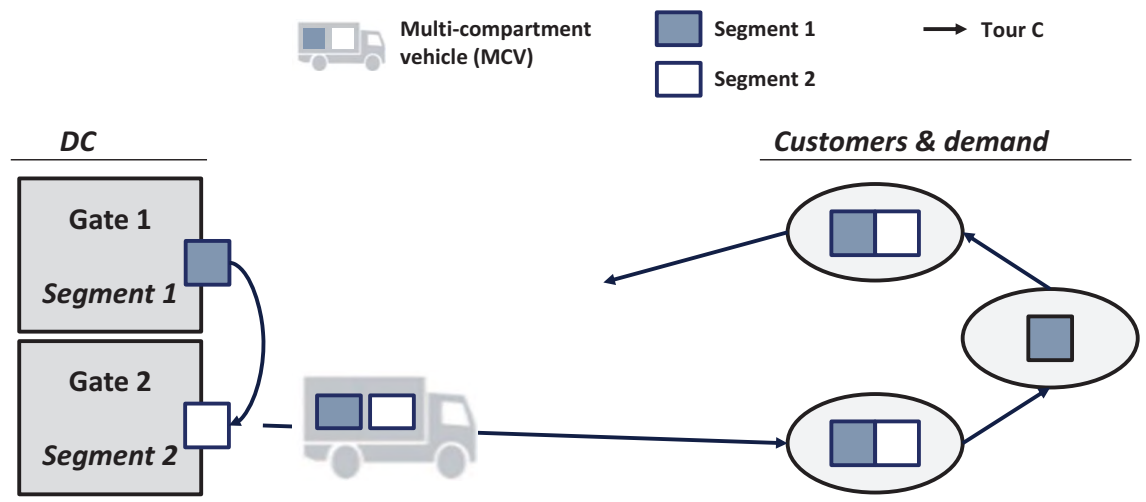

Figure 6: Distribution Process for MCVs

At the other end of the distribution, the supply of customers, the delivery of multiple segment enables retailers to combine several orders of the same customer. By doing this, a more efficient distribution can be achieved as tours across segments can be combined. Additionally, if a customer only has to be approached by one vehicle, service times decrease significantly in a total perspective. The overall distribution with MCVs is given in Figure 6. It shows the afore mentioned loading at several loading gates at the DC with an example of two segments and the joint delivery of those.

The consideration of MCVs shows that there are major advantages in using this new kind of vehicles but that also significant differences in the distribution process are involved. These differences lead to new and additional planning tasks for the retailers that have to be taken into 
account for the routing and therefore need to be integrated into the VRPs for MCVs. A starting point for a more efficient routing with MCVs is the identification and quantification of the additional loading process as well as the potential savings for bundling deliveries across multiple segments. The setup of more than one compartment on the loading area requires an advanced planning of the load carrying for transportation. Further, retailers have the option to choose between SCVs and MCVs and therefore it should be evaluated in which situation the one or the other is the better choice. These and further research topics are addressed in current literature and will be presented in the remainder of this article on the use of MCV in grocery distribution.

\section{Current Research}

In this section current research on the use of MCVs for the distribution of groceries is presented. A short overview on related publications in the field of MCVRPs is given before current literature for the application of MCVs in grocery distribution is discussed.

In general, literature on MCVRPS can be divided in applications with flexible and fixed compartment. The difference is, that in case of fixed compartments, the number and size of compartments on each vehicle is already given as input to the decision model. Flexible compartments on the other hand, can be adjusted flexible dependent on the loaded goods of each product segment. Fixed compartments are usually used for the transportation of petrol (e.g., Avella et al. [2004]) or other fluids/chemicals that may not be mixed during transportation. In our application we focus on flexible compartments and therefore only publications in this field are presented.

A first comprehensive model that uses flexible compartments for the distribution of both food and fuel was presented by Derigs et al. [2011]. They introduce a solver suite including a large neighborhood search (LNS), a local search and several construction heuristics within an adaptive search procedure. Their approach has been tested on various benchmark instances and provides further insights into the performance of different operators for the search. In a wider sense, Chajakis and Guignard [2003] consider the distribution of groceries for convenient stores. However, instead of real compartments the delivery of frozen or fresh goods is treated with cooling boxes or bulkheads. Henke et al. [2015b] present an MCVRP for the collection of glass waste. Their formulation considers flexible compartment sizes, but the variation in size is limited to predefined steps. This means that compartments are not fully flexible but can vary between given sizes. They developed a variable neighborhood search to solve the corresponding problem. Henke et al. [2015a] develop a branch-and-cut approach to this problem. Further, Koch et al. [2016] present a genetic algorithm for a similar problem setting to collect multiple products from customers. 
The focus of this article is on literature that concerns the distribution of groceries with MCVs and therefore publications in this field of research are presented in detail. We start with literature that deals with the basic influences of MCVs on the distribution, i.e., (i) the impact of additional processes involved and its effect on costs as well as (ii) the loading layout of a vehicle due to multiple compartments and the resulting restrictions for loading actions. The differences for the use of MCVs in distribution are connected to realistic process costs and the impact on the routing decisions are explained. Further, an extended decision model for the selection of SCVs and MCVs for the distribution fleet is presented that aims to identify the optimal fleet mix for the distribution fleet of a retailer. Lastly, we present an extension of the routing of MCVs for multiple periods. Here, the focus lies on customer satisfaction which can be improved if individual segments are delivered consistently over the complete planning horizon. Table 1 provides an overview of current research and the underlying projects discussed in this article.

\begin{tabular}{cll}
\hline Subsection & Problem Specification & Literature \\
\hline 2.1 & $\begin{array}{l}\text { Integration of loading and unloading costs for MCVs into } \\
\text { the decision model }\end{array}$ & Hübner and Ostermeier [2018] \\
2.2 & $\begin{array}{l}\text { Integration of loading constraints that have to be consid- } \\
\text { ered for MCVs }\end{array}$ & Ostermeier and Hübner [2017] \\
2.3 & $\begin{array}{l}\text { Selection of SCVs and MCVs for an optimal fleet mix for } \\
\text { distribution }\end{array}$ & Ostermeier et al. [2017] \\
2.4 & $\begin{array}{l}\text { Introduction of consistent deliveries over multiple seg- } \\
\text { ments with MCVs }\end{array}$ & Martins et al. [2017] \\
\hline
\end{tabular}

Table 1: Overview of current literature

\subsection{Loading $\&$ Unloading Costs for MCVs}

The central aspect when considering MCVs for grocery distribution are the arising additional processes, namely the loading and unloading. In Hübner and Ostermeier [2018], the authors analyze these processes and derive costs for the loading and unloading of MCVs. The costs are based on a case study with a major German retailer who uses MCVs for its own distribution fleet. The study comprises a time-and-motion study in which several tours of the retailer have been accompanied to collect the required data. In the following, the need to consider loading and unloading costs are presented and the main findings of the mentioned research is given.

Loading Costs. The higher loading costs for using MCVs are induced by the approach of multiple loading gates at the DC. The loading process is therefore more costly if MCVs are used compared to the use of SCVs where only one loading gate is approached for each tour. The loading costs of MCVs thereby depend on the number of segments that are loaded and therefore on the number of compartments used. The more different segments are loaded, the more compartments are needed 
and, consequently, the higher are the costs. The loading process is also illustrated in Figure 7 . It shows the situation at the DC when MCVs or SCVs are used for transportation. In the example, we assume that there are four segments available at the corresponding DC and the MCV tour comprises all four segments.

\section{Loading process with MCVs}

Example of MCV tour with four segments

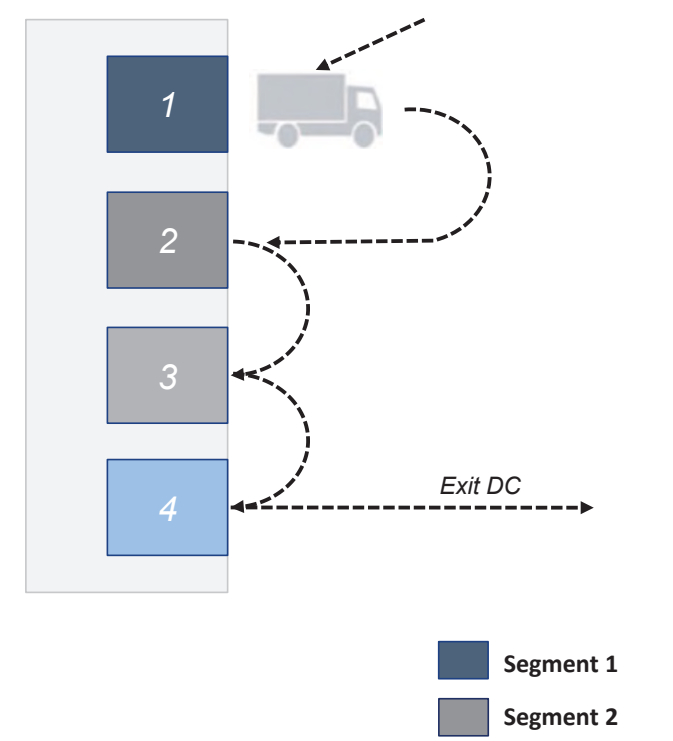

Loading process with SCVs

Example of SCV tour - only one segment can be loaded per tour

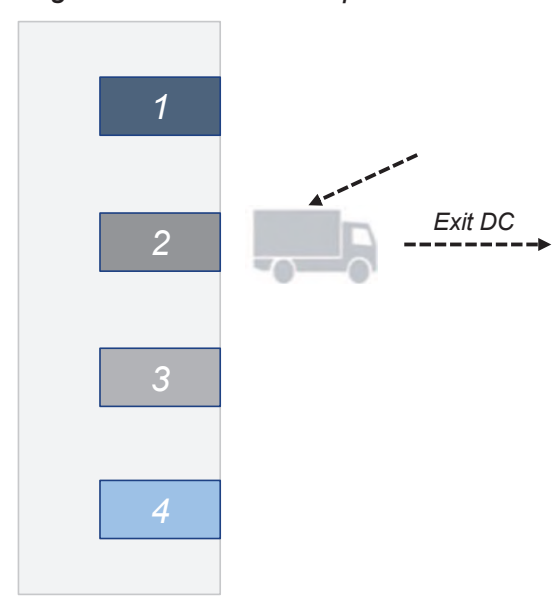

Segment 3

Segment 4

Figure 7: Loading process for SCVs and MCVs

Generally, for the loading aspect of the distribution we have to decide if it is beneficial to combine multiple segments on an MCV and approach several loading gates with one vehicle or if it is better to plan single segment tours with SCVs.

Unloading Costs. In contrast to the loading, the unloading processes are truncated when MCVs are used. As an MCV is able to combine different segments on the same tour, the joint supply of customers with multiple segments is possible. This means, that a customer who would usually receive a single delivery for each segment only needs to be approached once if the orders are combined on the same vehicle. This merging of different deliveries saves customer stops and opens up new options for the route building, leading to a potential reduction in transportation kilometers. An example of the contrast in the delivery with MCVs or SCVs is given in Figure 8 MCVs therefore enable a cost reduction when unloading processes are considered.

Altogether the use of MCVs implies a trade-off between higher loading costs and lower unloading costs by vehicle used and these costs need to be evaluated for the routing decisions. Therefore, loading and unloading costs are integrated in the objective function of the corresponding decision 
Example of MCV delivery for a customer with orders for four segments

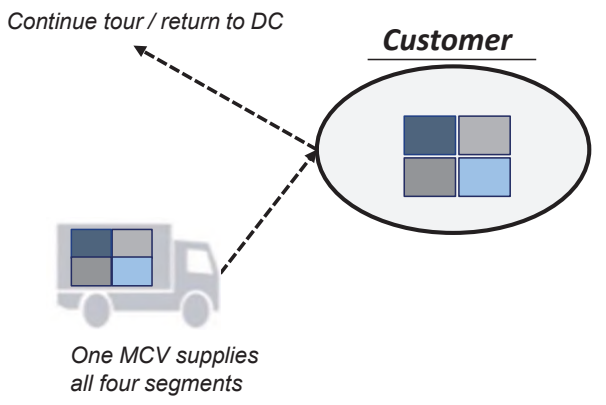

\section{Unloading process with SCVs}

Example of SCV deliveries for a customer with orders for four segments

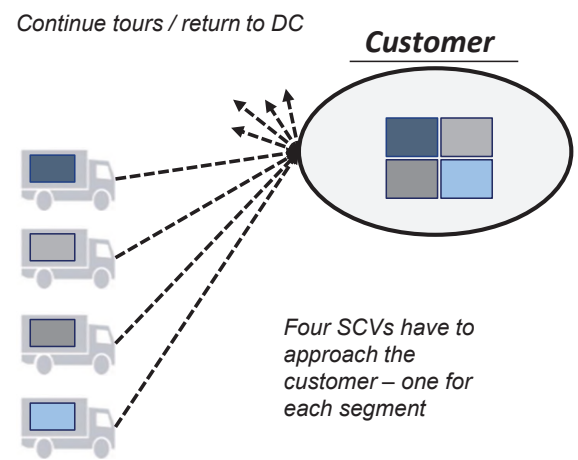

Segment 3

Segment 4

Figure 8: Unloading process for SCVs and MCVs

model. With this, the use of MCVs for distribution can be analyzed and also compared to the use of SCVs. The applied tests and findings on the research by Hübner and Ostermeier [2018] are summarized in the next paragraph.

Findings. Hübner and Ostermeier [2018] develop an LNS that uses operators that have shown very good results on other MCVRP instances as shown in other publications (Derigs et al. [2011]). Analyzing the impact of loading and unloading costs for MCVs on the routing decisions reveals several characteristics that influence the solution finding. The analyses comprise numerical tests with randomly generated data as well as data from a case study with a major German retailer. The tests aim to identify potential savings for a model that considers loading and unloading costs compared to a model that does not take into account these additional costs for MCVs. First of all, the number of available segments at the DC is a crucial diver for a potential cost saving if loading and unloading costs are integrated into cost evaluation. If more segments are available, more segments can be combined on an MCV and therefore it becomes more important to consider related costs. Second, the order size is decisive as in the case of small order sizes, more orders are combined on the same truck and thus, more compartments are used. Next, the distances between customer play a fundamental role for the effect of considering loading and unloading costs. For urban areas with short distances between customer, transportation costs are relatively low compared the arising loading and unloading costs. In this way the share of those costs in the solution is higher and their evaluation becomes more important. Lastly, the order structure of customer plays a central part 
for the cost consideration. If most customers order multiple segments it is more likely that their orders will be combined on a tour. Contrary, if the better part of customer only place one order, it is more likely that different orders for the same segment are combined on a vehicle instead of multiple segments. The key findings are also summarized in Table 2

\begin{tabular}{l|ll}
\hline Characteristic & Savings potential of & orrelation with increasing \\
& MCVRP_LU vs. MCVRP* & $\begin{array}{l}\text { Corres } \\
\text { loading \& unloading costs }\end{array}$ \\
\hline Product segments & more product segments & positive \\
\hline$\varnothing$ order size & lower average order size & $\begin{array}{l}\text { positive for order sizes } \geq 5 ; \\
\text { negative for order sizes }<5\end{array}$ \\
\hline Distance & shorter distances & positive \\
\hline $\begin{array}{l}\text { Number of orders } \\
\text { per customer }\end{array}$ & $\begin{array}{l}\text { more heterogeneous order } \\
\text { tures across customers }\end{array}$ & positive \\
\hline
\end{tabular}

Table 2: Findings for the development of saving potential

Additionally, a study with the afore mentioned retailer was carried out to compare the use of MCVs to the use of SCVs. The study shows that the use of MCVs offers a significant saving potential compared to the use of SCVs for the distribution of groceries. For the study, one example week of the retailer was considered. This includes six delivery days from one central DC to the corresponding customer. In total, a potential yearly saving in costs of around $6.5 \%$ is given for the considered DC.

\subsection{Loading Restrictions for $M C V s$}

Besides the involved additional processes and therefore costs for the use of MCVs, the loading situation itself is completely different compared to SCVs. The vehicle setup with different compartment leads to various options for the actual positioning of orders and compartments. This results in different layouts for exactly the same load and tour. In Figure 1 this situation is illustrated. It shows three different tours and for each tour two layout options.

Loading Constraints. While the loading of an SCV only requires the consideration of the correct sequence of customers on the tour, the loading of an MCV requires the collection of different segments and therefore their combination on the vehicle, the building of multiple compartments and the correct order of both customers and segments. The segment sequence is thereby the order of the different compartments (each dedicated to exactly one segment) on a truck. Here we have to make sure, that a customer order is not assigned to a compartment at the front of a truck (i.e., the driver end and therefore only due for unloading at the end of the tour) when it needs to be unloaded early on the corresponding tour. At the same time, the position and therefore setup of 


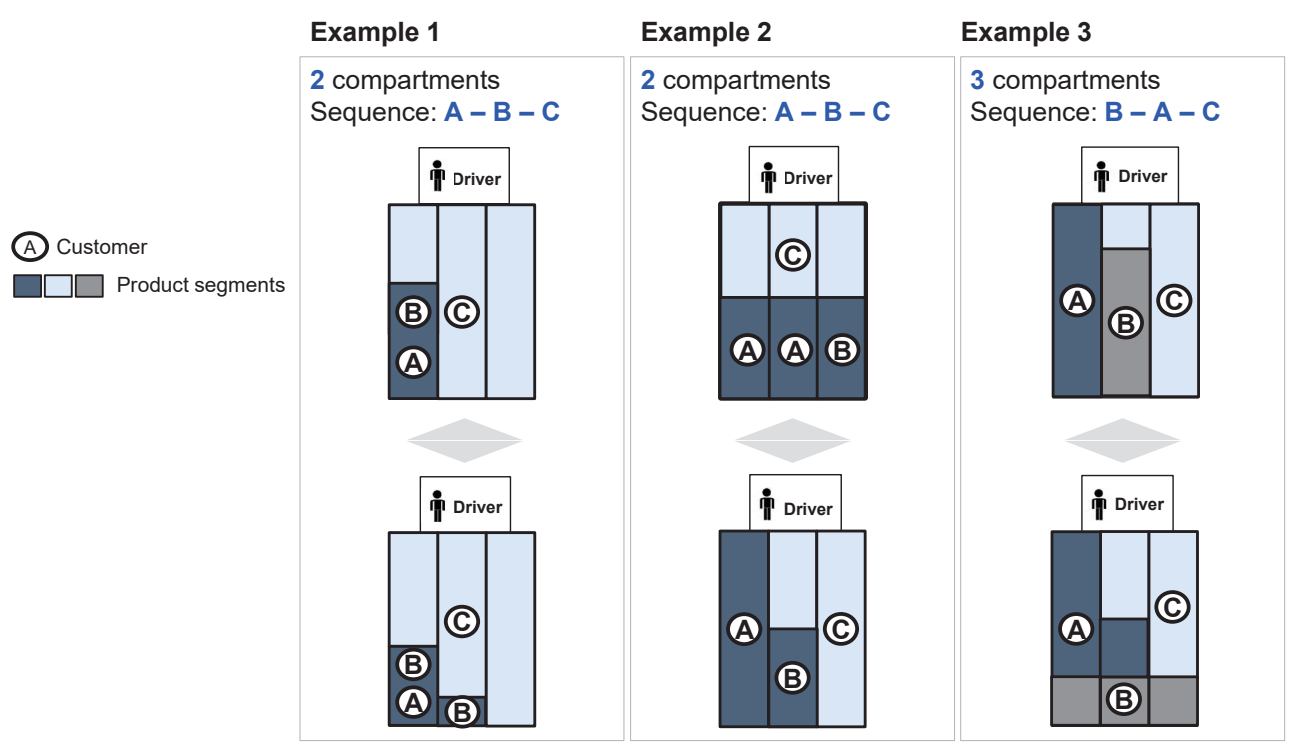

Figure 9: Examples for different loading layouts considering same orders and route

each compartment is fixed after the corresponding segment has been loaded completely. Thereby it is important to note, that each loading gate can only be approached once within the loading process of one tour and hence each segment has to be loaded completely with this stop a the gate. This means, that once a segment is loaded, the correspoding compartment setting is fixed and no alterations are possible during the further loading process as this would lead to a significant increase in loading times and costs. An example of a vehicle layout causing this kind of loading problem is given in Figure 10

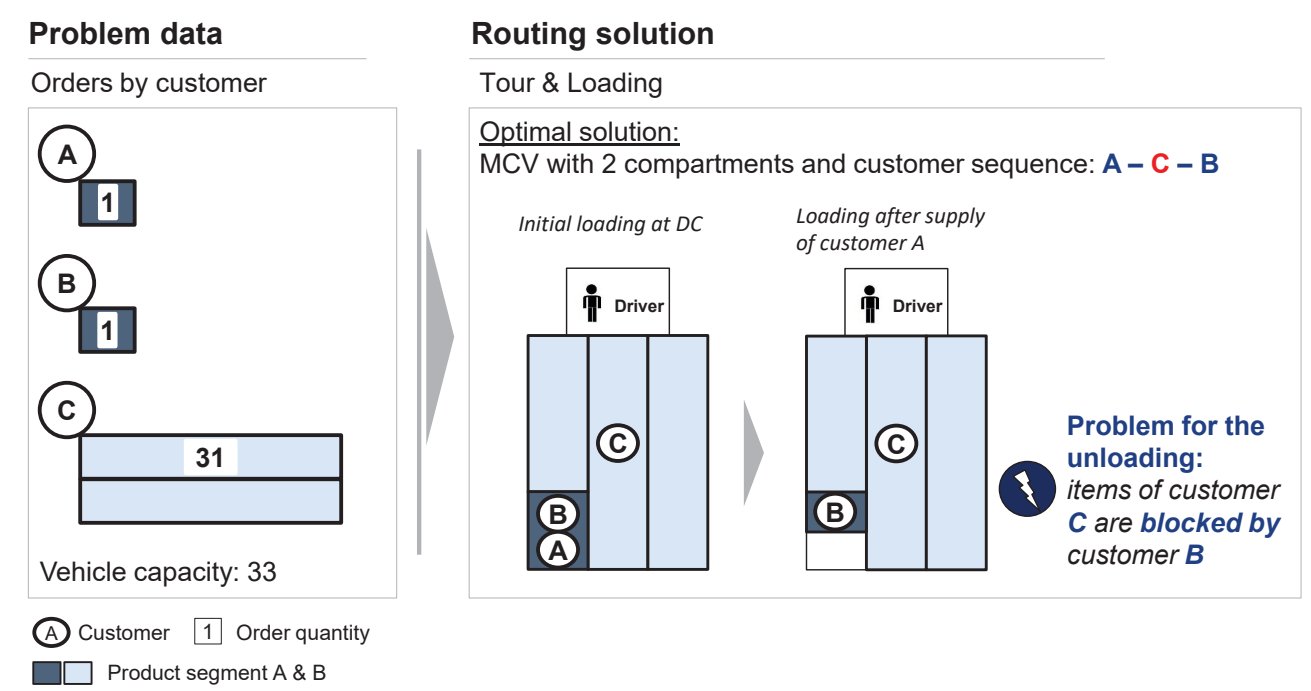

Figure 10: Examples for different loading layouts considering same orders and route

In this example, the driver would face an unacceptable unloading situation at customer C. To overcome such a situation, a driver would be forced to remove (large) parts of the load to rearrange 
orders for unloading. This is not allowed due to time and space restrictions for the delivery and due to transportation restrictions for the supply of temperature sensitive goods. As a consequence, it is necessary to include these loading constraints in the route building and thus in the MCVRP. In this way, additional constraints for the load building are considered during the search and only tours are excepted for the final schedule that adhere loading criteria for MCVs.

Findings. The authors show in their work that the consideration of loading constraints is a relevant problem in practice. An specialized LNS is developed that sequentially solves the routing and loading aspect of the problem. The loading issues are thereby addressed by a loading model that checks every tour for loading feasibility and removes orders that do violate the loading constraints. In extensive numerical experiments they analyze the introduction of loading constraints and show that as a general rule, the consideration of loading constraints leads to a minor increase in overall costs only but at the same time guarantees a barrier free unloading situation. They present two approaches to tackle the arising MCVRP with loading constraints to study the performance and effectiveness of their evaluation. They show that including the constraints into the search is more efficient than a mere ex post modification of the final routing solution. The integrated search requires longer computational times but is superior with respect to solution quality. The main drivers for the appearance of the described loading issues are the number of segments available and the order structure of customers (i.e., how many different segments are ordered per customer). The more segments are ordered, the higher is the need to incorporate loading constraints. In cases with four segments up to $100 \%$ of tours would lead to an unacceptable loading situation if no loading constraints are considered. Further, the relevance for the use in practice was shown in cooperation with a Portuguese grocery retailer.

\subsection{Fleet Mix}

So far, we considered the advantages and characteristics for SCVs and MCVs for their use in grocery distribution. It was always assumed that either MCVs or SCVs are used for the supply of customers. However, for retailers the most promising fleet is one with a mix of both vehicle types. In this way, the advantages of both vehicle types can be used to full capacity and therefore the most efficient distribution can be achieved.

Vehicle Selection. The optimal fleet and therefore the vehicle selection for grocery distribution depends on the evaluation of the available vehicle types. Subject to the requirements of each tour, i.e., the combination of different segments or the mere delivery of a single one, an SCV or MCV is chosen for transportation. The correct choice of a vehicle is therefore dependent on the costs 
implied by the selected type. In line with the previously presented work by Hübner and Ostermeier [2018] we already had a detailed look at the loading and unloading costs involved for MCVs. In the same way, the costs for SCVs are evaluated and integrated into the model. Additionally, the vehicle specific transportation costs need to be taken into account for the vehicle selection.

The transportation costs comprise different cost factors that are different between SCVs and MCVs. First of all, this involves procurement costs which are higher for MCVs due to their special technical features. Further, costs for insurance and maintenance can be considered as fixed costs dependent on the vehicle type. Based on these fixed costs Ostermeier et al. [2017] propose a vehiclespecific transportation cost rate per distance unit. These vehicle-dependent transportation costs comprise the described fixed costs plus variable costs for fuel and drivers. In this way, transportation costs dependent on the used vehicle type and traveled distances are included in the decision finding. With this, the MCVRP model is extended to select the best vehicle type for each route and thus results in a routing solution that also defines the best fleet mix for distribution. In a nutshell, the costs given in Figure 11 are taken into account for the routing and fleet mix decision.

\begin{tabular}{|c|c|c|}
\hline $\begin{array}{l}\text { Loading } \\
\text { Costs }\end{array}$ & $\begin{array}{c}\text { Transportation } \\
\text { Costs }\end{array}$ & $\begin{array}{l}\text { Unloading } \\
\text { Costs }\end{array}$ \\
\hline $\begin{array}{l}\text { Different loading } \\
\text { costs for SCVs and } \\
\text { MCVs } \\
\text { - Higher MCV costs } \\
\text { due to approach of } \\
\text { different loading } \\
\text { gates } \\
\text { MCV costs depend } \\
\text { on number of } \\
\text { loaded segments }\end{array}$ & 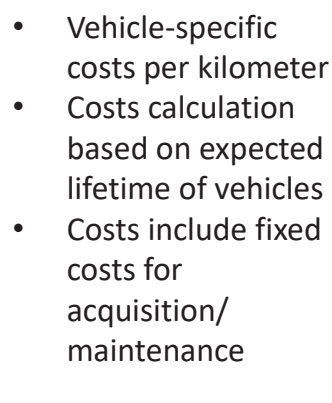 & $\begin{array}{l}\text { - Occur with every } \\
\text { stop at a customer } \\
\text { MCV costs are } \\
\text { higher than SCV } \\
\text { costs due to } \\
\text { additional } \\
\text { processes }\end{array}$ \\
\hline
\end{tabular}

Figure 11: Overview of decision relevant costs for the vehicle selection

To further illuminate the correlation between costs and vehicle types, Table 3 summarizes the differences in costs between the two vehicle types.

\begin{tabular}{|c|c|c|c|c|}
\hline \multirow[t]{2}{*}{ Vehicle type } & \multirow[t]{2}{*}{ Loading } & \multicolumn{2}{|c|}{ Transportation } & \multirow[t]{2}{*}{ Unloading } \\
\hline & & costs per $\mathrm{km}$ & travel distance & \\
\hline $\mathrm{SCV}$ & - & - & + & + \\
\hline $\mathrm{MCV}$ & + & + & - & - \\
\hline
\end{tabular}

Table 3: Schematic overview of cost differences between SCVs and MCVs (cf. Ostermeier and Hübner [2017])

Findings. The integration of vehicle selection between SCVs and MCVs was analyzed in Ostermeier and Hübner [2017] for different scenarios, mapping both settings in favor of SCVs and those in favor 
of MCVs. The authors used the LNS framework by Hübner and Ostermeier [2018] to solve the corresponding problem. The experiments comprise test instances for urban (i.e., shorter distances between customers) and rural (i.e., longer distances between customers) areas. Further, different settings for order size and structure have been analyzed. The authors show that a mixed fleet can reduce costs by up to $30 \%$ if compared to an SCV fleet and by up to $2 \%$ if the distribution was carried out by MCVs exclusively. In all test instances the best solution was provided by a mixed fleet and therefore shows the requirement of combining both SCVs and MCVs for distribution. The overall results of the tests can be summarized by the following statements.

- A mixed fleet is in all cases the best option for retailers as it uses the advantages of both vehicle types.

- Especially for delivery areas with larger distances between customers (rural) and higher order quantities, a mixed fleet allows significant cost savings.

- For order structures with only a few orders per customer, i.e. if a customer orders not all but only one or two of the available segments, it is important to combine both single and multi segment deliveries to save costs.

\subsection{Consistent Deliveries}

The last research presented in this article extends the MCVRP to multiple periods and therefore aims to produce a delivery schedule for a complete planning horizon. In the discussed application this comprises one delivery week of a retailer, i.e., up to 7 consecutive delivery days. For this planning horizon the routing decisions are considered simultaneously. In particular the presented research explores the introduction of consistent deliveries throughout the planning horizon for individual segments. A consistent delivery in this context means that for each customer the delivery for a particular segment happens at approximately the same time during the planning horizon. Consistent deliveries are particularly important for stores. Each store aligns its operations based on the deliveries of new products. The delivery process binds valuable resources from stores like workforce and storage. As a consequence, stores prefer to be supplied within a predefined time each day, i.e., within so called time-windows. In this way, the planning situation for stores is simplified and their resources can be used efficiently. Figure 10 motivates why consistent deliveries are need in grocery distribution.

Consistency Requirements. Consistent routing problems are already known for other VRP variants. Usually, the consistency attribute is used to increase customer satisfaction. The most common 

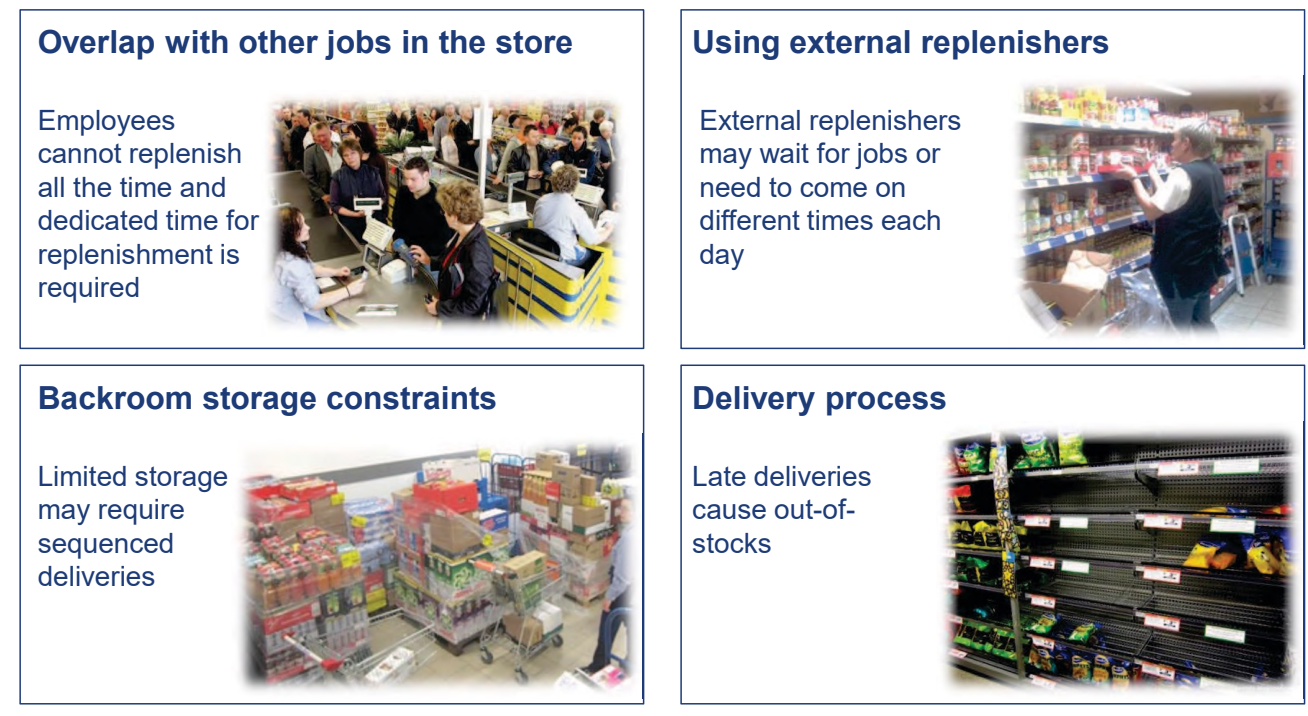

Figure 12: Stores require consistent deliveries to align in-store operations

specification for consistency are driver consistency that aims to assign each customer only to one or a limited number of drivers or tours and delivery consistency as it is discussed in our application. Having a closer look at other VRP variants with consistency, it is easy to spot that only a consistent delivery per customer is taken into account. In grocery distribution however, multiple orders need to be delivered to each customer and therefore the question arises which segments should be delivered at the same time or if different segments should be supplied at different times of the day. This question is addressed in the product-oriented time-window assignment MCVRP (PSTA-MCVRP) by Martins et al. [2017]. In their work, they assign a specific time-window to each segment of a customer and use the assigned time-window throughout the whole delivery week. In this way it is decided at what time each segment arrives and, moreover, which segment deliveries are combined. An example for the introduction of a consistent delivery is given in Figure 13. For a given example data set it shows a possible delivery schedule with and without consistency.

Findings. The consideration of multiple periods for an MCVRP further increases the already high complexity of the problem. Martins et al. [2017] therefore present a specialized heuristic that focuses on both routing and consistent deliveries. The presented heuristic, an adaptive LNS (ALNS) uses daily operators to find the best routing solution for each day, combined with weekly operators that focus on the alignment of the delivery times (consistency). The authors show the effect of introducing product-specific time windows and a consistent delivery schedule for different scenarios. Assuming penalty costs for earlier and/or later deliveries, they show that the overall costs for the complete period can be reduced if the whole planning horizon is regarded instead of solving each day separately. Further, the authors examine more restricted scenarios that map requirements in 


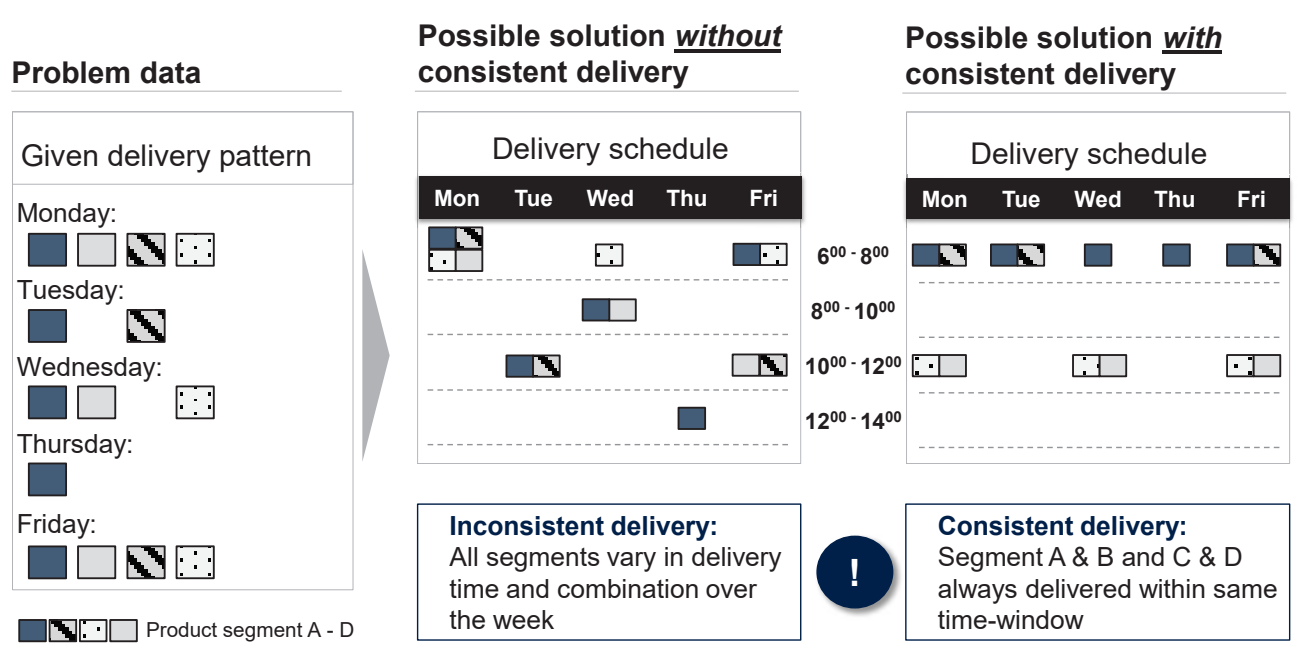

Figure 13: Impact of consistent deliveries - an exemplary delivery schedule

practice. For instance, stores require fresh products early in the morning to guarantee freshness and availability and therefore corresponding time-windows that are available for the fresh segment are restricted. They show, that in more restricted scenarios, the search tends to produce schedules with more time-windows per customer (i.e., moe segments are delivered separately) and less ontime deliveries (i.e., deliveries outside the assigned time-window). In this way, routing costs, and hence, overall costs are only slightly increased.

\section{Outlook}

The distribution of groceries poses many challenges for retailers and, in line with this, for researchers in this area. This article offers an introduction to the given problem specifications and describe the influence, use and impact on the distribution when MCVs are integrated. Based on the new challenges posed by MCVs we consider needed extensions of the decision problem. The extensions of the basic MCVRP are necessary to achieve a more realistic evaluation of costs and to integrate further aspects from practice. The current research comprises (i) the integration of loading and unloading costs, (ii) the consideration of loading constraints for MCVs and (iii) the vehicle selection for a mixed fleet of SCVs and MCVs. Furthermore, a work on the extension for multiple periods is presented to (iv) achieve a consistent delivery across segments for a complete planning horizon.

Of course, research does not end here. There are various challenges that still need to be addressed in literature and practice. One possible next step could be to extend the problem to a heterogeneous fleet with differing vehicle capacities. Next, for numerous VRP variants there are extensions with stochastic and varying demand. This is also a worthwhile path for MCVRPs 
in grocery distribution as retailers face a high fluctuation in d emand a nd s easonality e ffects. As MCVs combine different customer orders on the same vehicle this offers new possibilities for order frequency of customers. A customer could receive orders more frequently as different segments are supplied simultaneously. The impact of combined orders on inventory and delivery patterns should therefore be examined. An altered delivery frequency and/or order sizes could also show significant impact on operations at the s tores. Further a pplications could a ddress the MCVRP for home deliveries of online orders and store orders (see e.g., Hübner et al. [2016b], Hübner et al. [2016a]) as this is an ever increasing branch of trade.

All variants of the MCVRP are NP-hard problems and therefore very hard to solve. For each problem exist various solution approaches and possible new approaches to address these problems. As a consequence, MCVRPs pose the challenge to develop and test new efficient solution approaches. This needs to be done to decrease the needed runtime to find a feasible solution as well as to further improve the solution quality. In this article we focus on current research and content ourselves with the described problem specifications. However, there a re a lot of different ways to solve MCVRP and therefore there are many further paths for research.

\section{Acknowledgments}

The research of the author is founded by the German Ministry of Education and Research and the Hanns Seidel Foundation.

\section{References}

Avella, P., Boccia, M., Sforza, A., 2004. Solving a fuel delivery problem by heuristic and exact approaches. European Journal of Operational Research 152, 170-179.

Chajakis, E.D., Guignard, M., 2003. Scheduling deliveries in vehicles with multiple compartments. Journal of Global Optimization 26, 43-78.

Derigs, U., Gottlieb, J., Kalkoff, J., Piesche, M., Rothlauf, F., Vogel, U., 2011. Vehicle routing with compartments: Applications, modelling and heuristics. OR Spectrum 33, 885-914.

Golden, B.L., Raghavan, S., Wasil, E.A., 2008. The Vehicle Routing Problem: Latest Advances and New Challenges. Operations Research/Computer Science Interfaces Series, Springer.

Henke, T., Speranza, M.G., Wäscher, G., 2015a. A branch-and-cut algorithm for the multi-compartment vehicle routing problem with flexible c ompartments izes. W orking P aper N o. 0 4/2017, Otto-von-Guericke-University Magdeburg .

Henke, T., Speranza, M.G., Wäscher, G., 2015b. The multi-compartment vehicle routing problem with flexible compartment sizes. European Journal of Operational Research 246, 730-743.

Hübner, A., Holzapfel, A., Kuhn, H., 2016a. Distribution systems in omni-channel retailing. Business Research 9, $255-296$.

Hübner, A., Ostermeier, M., 2018. A multi-compartment vehicle routing problem with loading and unloading costs. Transportation Science, forthcoming.

Hübner, A., Wollenburg, J., Holzapfel, A., 2016b. Retail logistics in the transition from multi-channel to omni-channel. International Journal of Physical Distribution \& Logistics Management 46, 562-583. 
Koch, H., Henke, T., Wäscher, G., 2016. A genetic algorithm for the multi-compartment vehicle routing problem with flexible compartment sizes. Working Paper No. 04/2016, Otto-von-Guericke-University Magdeburg .

Laporte, G., 2009. Fifty years of vehicle routing. Transportation Science 43, 408-416.

Martins, S., Ostermeier, M., Amorim, P., Hübner, A., 2017. A multi-compartment vehicle routing problem with product-specific time-window assignment. Working Paper .

Ostermeier, M., Hübner, A., 2017. Vehicle selection for a multi-compartment vehicle routing problem. Working Paper

Ostermeier, M., Martins, S., Amorim, P., Hübner, A., 2017. Loading constraints for a multi-compartment vehicle routing problem. Working Paper .

Toth, P., Vigo, D., 2014. Vehicle Routing: Problems, Methods, and Applications, Second Edition. MOS-SIAM Series on Optimization, Society for Industrial and Applied Mathematics. 